\title{
Correction: Wai, M.G.C., et al. A Review of Pinealectomy-Induced Melatonin-Deficient Animal Models for the Study of Etiopathogenesis of Adolescent Idiopathic Scoliosis. Int. J. Mol. Sci. 2014, 15, 16484-16499
}

Gene Chi Wai Man ${ }^{1}$, William Wei Jun Wang ${ }^{2,3}$, Annie Po Yee Yim ${ }^{4}$, Jack Ho Wong ${ }^{5}$, Tzi Bun Ng ${ }^{5}$, Tsz Ping Lam ${ }^{4}$, Simon Kwong Man Lee ${ }^{6}$, Bobby Kin Wah Ng ${ }^{4}$, Chi Chiu Wang ${ }^{1}$, Yong Qiu ${ }^{2,3}$ and Jack Chun Yiu Cheng ${ }^{3,4, *}$

1 Department of Obstetrics and Gynaecology, Faculty of Medicine, The Chinese University of Hong Kong, Hong Kong, China;

E-Mails: geneman@cuhk.edu.hk (G.C.W.M.); ccwang@cuhk.edu.hk (C.C.W.)

2 Department of Spine Surgery, Drum Tower Hospital, Nanjing University Medical School, Nanjing 210008, China; E-Mails: drwilliamwang@163.com (W.W.J.W.); scoliosis2002@sina.com (Y.Q.)

3 Joint Scoliosis Research Center of the Chinese University of Hong Kong and Nanjing University, Hong Kong, China

4 Department of Orthopaedics and Traumatology, Faculty of Medicine,

The Chinese University of Hong Kong, Hong Kong, China;

E-Mails: anniepym@gmail.com (A.P.Y.Y.); tplam@ort.cuhk.edu.hk (T.P.L.); bobng@ort.cuhk.edu.hk (B.K.W.N.)

5 School of Biomedical Sciences, Faculty of Medicine, The Chinese University of Hong Kong, Hong Kong, China; E-Mails: jack1993@yahoo.com (J.H.W.); tzibunng@cuhk.edu.hk (T.B.N.)

6 Lee Hysan Clinical Research Laboratory, Faculty of Medicine, The Chinese University of Hong Kong, Hong Kong, China; E-Mail: simonlee@cuhk.edu.hk

* Author to whom correspondence should be addressed; E-Mail: jackcheng@cuhk.edu.hk; Tel.: +852-26-098-631; Fax: +852-26-036-889.

Received: 2 January 2015 / Accepted: 10 January 2015 / Published: 29 January 2015

The authors wish to make the following corrections to this paper [1]:

The first name and surname of the authors were reversed. It should be corrected in the following format (with the surname in bold text): 
Gene Chi Wai Man, William Wei Jun Wang, Annie Po Yee Yim, Jack Ho Wong, Tzi Bun Ng, Tsz Ping Lam, Simon Kwong Man Lee, Bobby Kin Wah Ng, Chi Chiu Wang, Yong Qiu and Jack Chun Yiu Cheng

The authors would like to apologize for any inconvenience caused to the readers by these changes.

\section{Reference}

1. Wai, M.G.C.; Jun, W.W.W.; Yee, Y.A.P.; Ho, W.J.; Bun, N.T.; Ping, L.T.; Man, L.S.K.; Wah, N.B.K.; Chiu, W.C.; Yong, Q.; et al. A Review of Pinealectomy-Induced Melatonin-Deficient Animal Models for the Study of Etiopathogenesis of Adolescent Idiopathic Scoliosis. Int. J. Mol. Sci. 2014, 15, 16484-16499.

(C) 2015 by the authors; licensee MDPI, Basel, Switzerland. This article is an open access article distributed under the terms and conditions of the Creative Commons Attribution license (http://creativecommons.org/licenses/by/4.0/). 